\title{
Evaluation of Conceptual Midcourse Guidance Laws for Long-Range Exoatmospheric Interceptors
}

\author{
Mohsen Dehghani Mohammad-abadi ${ }^{1}$, Seyed Hamid Jalali-Naini ${ }^{1}$
}

\begin{abstract}
This paper presents a comprehensive study on the performance analysis of 8 conceptual guidance laws for exoatmospheric interception of ballistic missiles. The problem is to find the effective thrust direction of interceptor for interception of short-to-super range ballistic missiles. The zero-effort miss and the generalized required velocity concept are utilized for interception of moving targets. By comparison of the 8 conceptual guidance laws, the thrust direction is suggested to be in the direction of generalized velocity-to-begained, or constant velocity-to-be-gained direction, rather than to be in the direction along zero-effort miss, or that of linear optimal solution for long-to-super range interception. Even for short coasting ranges, the generalized velocity-to-be-gained may be utilized because of reasonable computational burden for required velocity rather than the numerical computation for zero-effort miss or linear optimal solution with the same miss distance error. In addition, the fuel consumption of the suggested direction has less sensitivity due to estimation error in intercept time. The guidance law based on constant velocity-to-be-gained direction and the optimal solution are suitable for satellites launch vehicles and space missions.
\end{abstract}

KEYWORDS: Exoatmospheric midcourse guidance, Effective thrust direction, Zero-effort miss, Velocity-to-be-gained, Long-range interceptor, Anti-ballistic guidance.

\section{INTRODUCTION}

Exoatmospheric intercept guidance improvements are of high interest in anti-ballistic air defense systems. The main subjects in this area are focused on midcourse and terminal phases of flight for anti-ballistic interceptors. The design considerations for the midcourse guidance are different from the terminal phase one. In the midcourse phase, the on-board trajectory optimization and trajectory shaping are the main issues, whereas the noise contamination and hit probability against very-high speed targets are the key issues for a terminal guidance law (Zarchan 2012).

The literature on exoatmospheric intercept guidance laws can be categorized into intercept guidance laws against moving targets and guidance laws for space missions including ballistic missiles. Since the concepts and guidance algorithms of ballistic missiles are similar to space vehicle guidance laws, we put them in the same category. The early literature on the subject of optimal 2-point guidance for interception of moving targets is based on zero-effort miss (ZEM) in flat-Earth model (Bryson and Ho 1975). In this case, the acceleration command in the optimal energy problems is obtained proportional to ZEM vector. Precisely speaking, the commanded acceleration of optimal energy guidance laws with final constraints in linear systems is obtained in the form of the predicted error vector pre-multiplied by a gain matrix. In the case that the final position vector is only constrained, the solution simplifies to ZEM vector pre-multiplied by a time-varying gain matrix (Rusnak and Meir 1991). In a special case, if the airframe and control systems are assumed to be identical for 3 axes, the matrix gain

1.Tarbiat Modares University - Faculty of Mechanical Engineering - Aerospace Group - Tehran/Tehran - Iran

Author for correspondence: Seyed Hamid Jalali-Naini | Tarbiat Modares University - Faculty of Mechanical Engineering - Jalal Al Ahmad Street, No 7 P.0. box14115-111 - Tehran/Tehran - Iran | E-mail: shjalalinaini@modares.ac.ir

Received: Jun. 06, 2016 | Accepted: Aug. 13, 2016 
simplifies to a scalar, i.e. the commanded acceleration becomes proportional to ZEM (Rusnak and Meir 1991). Two classes of explicit guidance laws based on ZEM have been developed with different assumptions for interceptor dynamics and types of target maneuvers (Jalali-Naini 2004).

In spherical-Earth model, in spite of the assumption of a perfect control system, the optimal maneuver is not obtained in the direction of ZEM because of the non-linear nature of the gravitational acceleration. Most literature on exoatmospheric intercept problems utilized the ZEM vector as an effective direction for thrust vectoring of the interceptor (Massoumnia 1995; Feng et al. 2009; Li et al. 2013). The ZEM can be approximated in an inverse-square gravity field (Li et al. 2013; Mohammad-abadi and Jalali-Naini 2016) or numerically computed on-board with a reasonable integration time step as in predictive guidance scheme (Zarchan 2012). As mentioned before, even ZEM is computed exactly in the spherical-Earth model; the acceleration command along the ZEM is not, mathematically, an optimal solution.

On the other hand, guidance laws for space missions are based on the concept of required velocity and velocity-to-begained (Battin 1999; Martin 1965, 1966). At a first glance, the concepts of the 2 guidance categories seem to be different. The concepts of required velocity and velocity-to-be-gained can also be utilized or generalized for interception of moving targets (Jalali-Naini and Pourtakdoust 2005; Chen et al. 2010). The velocity-to-be-gained vector becomes proportional to ZEM when the gravitational acceleration is assumed to be constant. In a linearized inverse square gravity field, the velocity-to-begained vector is obtained in the form of ZEM pre-multiplied by a time-varying gain matrix (Jalali-Naini and Pourtakdoust 2007). Both ZEM and required velocity can be calculated for a linearized gravity field. Several solutions have been presented using a linearized gravity with different assumptions as treated by Newman (1996) and Deihoul and Massoumnia (2003) for interception of ballistic missiles. The ZEM was also obtained for a linear gravity considering control system dynamics and target maneuvers (Jalali-Naini 2008).

Several anti-ballistic guidance schemes were presented based on ZEM, as mentioned earlier. In these guidance schemes, the corrective maneuver is applied in the direction proportional to ZEM, but the guidance gain is modified, manipulated, and/or theoretically or empirically designed. For space missions, Battin (1999) introduced a guidance scheme in order to keep the direction of velocity-to-be-gained constant, and Sokkappa
(1966) obtained an approximate optimal guidance assuming Q-matrix to be constant. Circi (2004) compared Battin's formula with the numerical optimal solution for a satellite launch vehicle. For short-range anti-ballistic interception, guidance laws based on ZEM perform well whereas for long-to-super range interception, the maneuvering direction needs to be modified to the direction of velocity-to-be-gained or possibly an optimal one. The question is: what direction should be utilized for what ranges. The present study focuses on quantifying the answer to this question, based on accuracy and some implementation issues. Fortunately, several efficient algorithms are available for on-board computation of required velocity and Q-matrix (Zarchan 2012; Arora et al. 2015; Ahn et al. 2015).

There is another type of guidance laws for space missions or interception in exoatmosphere, referred to as General Energy Management (GEM) for solid rocket motors without cut-off capability (Zarchan 2012). In this class of guidance schemes, the maneuvering direction is somewhat deviated from a desired direction, ZEM or velocity-to-be-gained, in order to manage the wasting of extra fuel of the rocket so as the space vehicle reaches the required velocity at burnout. Since our study focuses on optimal energy guidance, GEM-type guidance schemes are beyond its scope.

In this article, the performance of the midcourse phase of exoatmospheric interceptors is compared for 8 conceptual guidance schemes. It is assumed that this midcourse phase is followed by a coasting phase. In other words, the interceptor is due to reach near the target position, coasting ballistically, where a small kinetic kill vehicle (KKV) is due to separate in order to intercept its target with minimum effort.

\section{BASIC FORMULATION}

The governing equations of motion for a particle $P$ (interceptor or target) with a given acceleration vector, $\mathbf{a}_{p}(t)$, are given by:

$$
\begin{aligned}
& \dot{\mathbf{r}}_{p}=\mathbf{v}_{p} \\
& \dot{\mathbf{v}}_{p}=\mathbf{a}_{p}(t)
\end{aligned}
$$

where: $\mathbf{r}_{p}, \mathbf{v}_{p}$, and a denote position, velocity, and acceleration vectors at current time $t$ in an inertial reference, respectively; the subscript $p$ also represents the particle $P$. 
The final position (at final time $t_{f}$ ) is obtained by integrating twice with respect to the time as follows:

$$
\mathbf{r}_{p}\left(t_{f}\right)=\mathbf{r}_{p}(t)+t_{g o} \mathbf{v}_{p}(t)+\int_{t}^{t_{f}} \int_{t}^{\xi} \mathbf{a}_{p}(\tau) d \tau d \xi
$$

where: $\tau$ and $\xi$ are dummy indices for time. By converting the preceding double integral to the single one, we have:

$$
\mathbf{r}_{p}\left(t_{f}\right)=\mathbf{r}_{p}(t)+t_{g o} \mathbf{v}_{p}(t)+\int_{t}^{t_{f}}\left(t_{f}-\xi\right) \mathbf{a}_{p}(\xi) d \xi
$$

where: $t_{g o}=t_{f}-t$ is the time-to-go until the final time.

In an exoatmospheric free-flight motion, we have $\mathbf{a}_{p}=\mathbf{G}_{p}$, where $\mathbf{G}_{p}$ is the gravitational acceleration, i.e. $\mathbf{G}_{p}=-\mu \mathbf{r}_{p} /\left|\mathbf{r}_{p}\right|^{3}$ for a spherical-Earth model, and $\mu$ is the Earth's gravitational constant. Therefore, Eq. 3 may be written in the following form:

$$
\mathbf{r}_{p}\left(t_{f}\right)=\mathbf{r}_{p}(t)+t_{g o} \mathbf{v}_{p}(t)+\int_{t}^{t_{f}}\left(t_{f}-\xi\right) \mathbf{G}_{p}\left[\mathbf{r}_{p}(\xi)\right] d \xi
$$

The preceding equation simplifies for a special case of constant gravity, that is,

$$
\mathbf{r}_{p}\left(t_{f}\right)=\mathbf{r}_{p}+t_{g o} \mathbf{v}_{p}+\frac{1}{2} \mathbf{G}_{p} t_{g o}^{2}
$$

The 3-D intercept geometry with respect to an inertial reference $(O x y z)$ is shown in Fig. 1, in which the interceptor $I$, having velocity $\mathbf{v}_{I}$, is pursuing its target $T$, with velocity $\mathbf{v}_{T}$. The interceptor and target position vectors are denoted by $\mathbf{r}_{I}$ and $\mathbf{r}_{T}$, respectively. The relative position $\mathbf{r}$ and velocity $\mathbf{v}$ for the interception problem are defined as:

$$
\begin{aligned}
& \mathbf{r}=\mathbf{r}_{T}-\mathbf{r}_{I} \\
& \mathbf{v}=\mathbf{v}_{T}-\mathbf{v}_{I}
\end{aligned}
$$

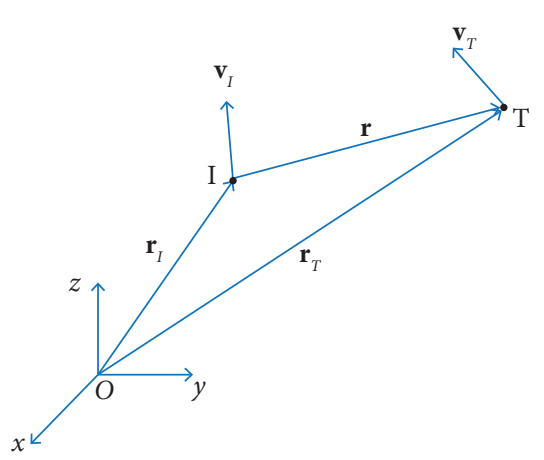

Figure 1. Engagement geometry.
The relative equations of motion are then given by:

$$
\begin{aligned}
& \dot{\mathbf{r}}=\mathbf{v} \\
& \dot{\mathbf{v}}=\mathbf{a}_{T}-\mathbf{a}_{I}
\end{aligned}
$$

where: $\mathbf{a}_{T}$ and $\mathbf{a}_{l}$ are the respective target and interceptor accelerations in inertial reference. Using Eqs. 3 and 6a, the final relative position is written as:

$$
\begin{aligned}
& \mathbf{r}\left(t_{f}\right)=\mathbf{r}_{T}\left(t_{f}\right)-\mathbf{r}_{I}\left(t_{f}\right)=\mathbf{r}_{T}(t)+t_{g o} \mathbf{v}_{T}(t)+ \\
& 1+\int_{t}^{t_{f}}\left(t_{f}-\xi\right) \mathbf{a}_{T}(\xi) d \xi-\mathbf{r}_{I}(t)-t_{g o} \mathbf{v}_{I}(t)-\int_{t}^{t_{f}}\left(t_{f}-\xi\right) \mathbf{a}_{I}(\xi) d \xi
\end{aligned}
$$

Therefore, the relative formulation for miss distance is expressed as follows:

$$
\mathbf{r}\left(t_{f}\right)=\mathbf{r}(t)+t_{g o} \mathbf{v}(t)+\int_{t}^{t_{f}}\left(t_{f}-\xi\right)\left[\mathbf{a}_{T}(\xi)-\mathbf{a}_{I}(\xi)\right] d \xi
$$

In an exoatmospheric free-flight motion, we have $\mathbf{a}_{T}=\mathbf{G}_{T}$ and $\mathbf{a}_{I}=\mathbf{G}_{I}$, and the substitution yields:

$$
\begin{aligned}
\mathbf{r}\left(t_{f}\right) & =\mathbf{r}(t)+t_{g o} \mathbf{v}(t)+ \\
& +\int_{t}^{t_{f}}\left(t_{f}-\xi\right)\left\{\mathbf{G}\left[\mathbf{r}_{T}(\xi)\right]-\mathbf{G}\left[\mathbf{r}_{I}(\xi)\right]\right\} d \xi
\end{aligned}
$$

The solution of the preceding equation is not straightforward. One approach to the problem is the linearization of its non-linear term, i.e. gravitational acceleration, that is,

$$
\mathbf{G}_{I}=\mathbf{G}_{T}+\mathbf{E}(t) \mathbf{r}
$$

where:

$$
\mathbf{E}(t)=-\left.\frac{\partial \mathbf{G}}{\partial \mathbf{r}_{I}}\right|_{\text {reference point }}, \mathbf{G}=\mathbf{G}_{T}-\mathbf{G}_{I}
$$

Therefore, the linearized state-space form for an exoatmospheric interceptor having thrust acceleration, $\mathbf{a}_{\mathrm{th}}\left(\mathbf{a}_{\mathrm{I}}=\mathbf{G}_{\mathrm{I}}+\mathbf{a}_{\mathrm{th}}\right)$, is obtained as (Newman 1996):

$$
\frac{d}{d t}\left[\begin{array}{l}
\mathbf{r} \\
\mathbf{v}
\end{array}\right]=\left[\begin{array}{cc}
\mathbf{0} & \mathbf{I} \\
-\mathbf{E}(t) & \mathbf{0}
\end{array}\right]\left[\begin{array}{l}
\mathbf{r} \\
\mathbf{v}
\end{array}\right]+\left[\begin{array}{c}
\mathbf{0} \\
-\mathbf{a}_{t h}
\end{array}\right]
$$

where: $\mathbf{I}$ is a $3 \times 3$ identity matrix, and

$$
\mathbf{E}=\frac{\mu}{R^{3}}\left[\mathbf{I}-3 \frac{\mathbf{R R}^{T}}{R^{2}}\right], \quad R=|\mathbf{R}|
$$


Several assumptions can be made for the parameter $\mathbf{R}$ such as $\mathbf{R}=\mathbf{r}_{I}\left(\right.$ Newman 1996) and $\mathbf{R}=\mathbf{r}_{T}, \mathbf{R}=\left(\mathbf{r}_{I}+\mathbf{r}_{T}\right) / 2, \mathbf{R}=\mathbf{r}_{T}\left(t_{f}\right)$ or $\left.\mathbf{R}=\mathbf{r}_{I}+\mathbf{r}_{T}+\mathbf{r}_{T}\left(t_{f}\right)\right) / 3$ as treated by Deihoul (2003). The author claimed that the last relation for $\mathbf{R}$ gives better results, so it is used in our comparison study.

The solution of the homogenous differential equation $(\ddot{\mathbf{r}}+\mathbf{E r}=\mathbf{0})$ is obtained in terms of the state-transition matrix, $\boldsymbol{\Phi}\left(t, t_{0}\right)$, as treated by Newman (1996) when $\mathbf{E}$ is assumed to be a constant matrix, that is,

$$
\mathbf{r}(t)=\Phi_{11}\left(t, t_{0}\right) \mathbf{r}\left(t_{0}\right)+\Phi_{12}\left(t, t_{0}\right) \mathbf{v}\left(t_{0}\right)
$$

where: $\Phi_{i j}$ is a $3 \times 3$ submatrix $(i, j=1,2)$ in the following partitioned matrix form:

$$
\boldsymbol{\Phi}\left(t, t_{0}\right)=\left[\begin{array}{ll}
\boldsymbol{\Phi}_{11} & \boldsymbol{\Phi}_{12} \\
\boldsymbol{\Phi}_{21} & \boldsymbol{\Phi}_{22}
\end{array}\right]
$$

and

$$
\begin{aligned}
& \boldsymbol{\Phi}_{11}=\boldsymbol{\Phi}_{22}=\mathbf{I}-\frac{1}{2 !} \mathbf{E}\left(t-t_{0}\right)^{2}+\frac{1}{4 !} \mathbf{E}^{2}\left(t-t_{0}\right)^{4}-\ldots \\
& \boldsymbol{\Phi}_{12}=\mathbf{I}\left(t-t_{0}\right)-\frac{1}{3 !} \mathbf{E}\left(t-t_{0}\right)^{3}+\frac{1}{5 !} \mathbf{E}^{2}\left(t-t_{0}\right)^{5}-\ldots \\
& \boldsymbol{\Phi}_{21}=-\mathbf{E}\left(t-t_{0}\right)+\frac{1}{3 !} \mathbf{E}^{2}\left(t-t_{0}\right)^{3}-\frac{1}{5 !} \mathbf{E}^{3}\left(t-t_{0}\right)^{5}+\ldots
\end{aligned}
$$

Therefore, the linearized relation of the miss distance vector in terms of the current states is given by:

$$
\mathbf{r}\left(t_{f}\right)=\boldsymbol{\Phi}_{11}\left(t_{f}, t\right) \mathbf{r}+\boldsymbol{\Phi}_{12}\left(t_{f}, t\right) \mathbf{v}
$$

\section{ZERO-EFFORT MISS}

The ZEM at the current time, $\operatorname{ZEM}(t)$, is the distance that the interceptor would miss its target position if the interceptor made no corrective maneuver $\left(\mathbf{a}_{t h}\right)$ after the time $t$ (Zarchan 2012), that is,

$$
\operatorname{ZEM}(t)=\mathbf{r}_{I}^{*}\left(t_{f}\right)-\left.\mathbf{r}_{I}\left(t_{f}\right)\right|_{\mathbf{a}_{t h}(\xi)=0 \text { for } \xi \geq t}
$$

where: $\mathbf{r}_{I}^{*}\left(t_{f}\right)$ is the desired final position of the interceptor.

For an exoatmospheric interceptor $\left(\mathbf{a}_{I}=\mathbf{G}_{I}+\mathbf{a}_{t h}\right)$, we have:

$$
\begin{aligned}
& \left.\mathbf{r}_{I}\left(t_{f}\right)\right|_{\mathbf{a}_{t h}(\xi)=\mathbf{0} \text { for } \xi \geq t}=\mathbf{r}_{I}+t_{g o} \mathbf{v}_{I}+ \\
& \quad+\int_{t}^{t_{f}}\left(t_{f}-\xi\right) \mathbf{G}\left[\left.\mathbf{r}_{I}(\xi)\right|_{a_{t h}=\mathbf{0}}\right] d \xi
\end{aligned}
$$

The substitution of Eq. 22 into Eq. 21 yields:

$$
\begin{aligned}
\operatorname{ZEM}(t)= & \mathbf{r}_{I}^{*}\left(t_{f}\right)-\mathbf{r}_{I}-t_{g o} \mathbf{v}_{I}-\int_{t}^{t_{f}}\left(t_{f}-\xi\right) \\
& \mathbf{G}\left[\left.\mathbf{r}_{I}(\xi)\right|_{\mathbf{a}_{t h}=0}\right] d \xi
\end{aligned}
$$

For a free-falling target, i.e. $\mathbf{a}_{T}=\mathbf{G}_{T}$, using Eq. 10, the ZEM relation can also be expressed in relative coordinates as follows:

$$
\begin{aligned}
& \operatorname{ZEM}(t)=\mathbf{r}+t_{g o} \mathbf{v}+\int_{t}^{t_{f}}\left(t_{f}-\xi\right) \\
&\left\{\mathbf{G}\left[\mathbf{r}_{T}(\xi)\right]-\mathbf{G}\left[\left.\mathbf{r}_{I}(\xi)\right|_{\mathbf{a}_{t h}=0}\right]\right\} d \xi
\end{aligned}
$$

where: $\mathbf{r}_{I}^{*}\left(t_{f}\right)=\mathbf{r}_{T}\left(t_{f}\right)$.

The preceding relation simplifies for a special case of constant gravity or for the case that the interceptor is assumed to be near its target as treated by Massoumnia (1995), that is,

$$
\operatorname{ZEM}(t)=\mathbf{r}+t_{g o} \mathbf{v}
$$

Using Eq. 20, the linearized ZEM relation is given by:

$$
\mathbf{Z E M}_{\text {Lin }}(t)=\Phi_{11}\left(t_{f}-t\right) \mathbf{r}+\Phi_{12}\left(t_{f}-t\right) \mathbf{v}
$$

Two different definitions of ZEM are utilized in the guidance theory. In basic definition, the ZEM is defined as a miss distance vector without further control effort. The intercept time is not imposed to the intercept problem and it is the time of the nearest distance between an interceptor and its target without further control effort. The second definition is based on a specified final time and comes from linear optimal guidance laws with the assumption of a fixed final time. The ZEM vector for the basic definition is, here, denoted by $\mathbf{Z E M}_{\text {min }}$ whereas it is denoted by ZEM for the second definition. The final time, the time of the nearest distance denoted by $t_{f_{\mathrm{ZEM}}^{*}}^{*}$, for the basic definition is obtained by $\partial|\mathbf{Z E M}| / \partial t_{f}=0$. For example, in a special case of constant gravity, from Eq. 25, we have:

$$
\frac{\partial|\mathbf{Z E M}|}{\partial t_{g o}}=0 \Rightarrow t_{g o_{\mathrm{ZEM}}}^{*}=-\frac{\mathbf{r} \cdot \mathbf{v}}{|\mathbf{v}|^{2}}
$$

where: $t_{{ }_{\text {go }} \text { ZEM }}=t_{f_{\mathrm{ZEM}}}^{*}-t$.

It is worth noting that the component of ZEM perpendicular to the interceptor-target line-of-sight (LOS), $\mathbf{Z E M}_{\text {PLOS }}$, may be replaced for ZEM in a guidance formulation as treated by Zarchan (2012). 


\section{GENERALIZED REQUIRED VELOCITY}

The required velocity, $\mathbf{v}_{R}$, for Lambert's problem is defined as an instantaneous velocity, required to satisfy the final position constraint in a specified final time (Battin 1999). This concept is well-known in space missions and surface-to-surface applications. The implementation of guidance laws based on the required velocity may be implicit or explicit.

The required velocity concept may be generalized for an intercept problem against a moving target in the endoatmosphere considering interceptor dynamics. The interceptor desired velocity $\mathbf{v}^{*}$ is the velocity that makes ZEM equal to zero. This desired velocity is referred to as generalized required velocity (Jalali-Naini and Pourtakdoust 2005). The interceptor dynamics is, here, assumed to be perfect, the interceptor moves in the exoatmosphere, and a moving target is considered. For brevity, we use the term "required velocity" instead of "generalized required velocity".

For example, the required velocity for the case of constant gravity is obtained from Eq. 23 as:

$$
\mathbf{v}_{R}=\frac{\mathbf{r}_{T}\left(t_{f}\right)-\mathbf{r}}{t_{g o}}-\frac{1}{2} \mathbf{G} t_{g o}
$$

Therefore, the relation between the required velocity and ZEM for the case of constant gravity is simply obtained as:

$$
\mathbf{Z E M}=\mathbf{v}_{g} t_{g o}
$$

where: $\mathbf{v}_{g}$ is referred to as the velocity-to-be-gained $\left(\mathbf{v}_{g}=\mathbf{v}_{R}-\mathbf{v}_{I}\right)$.

For a spherical-Earth model, the required velocity causes Eq. 23 equal to zero, that is,

$$
\begin{aligned}
\mathbf{0} & =\mathbf{r}_{T}\left(t_{f}\right)-\mathbf{r}_{I}-t_{g o} \mathbf{v}_{R}- \\
& -\int_{t}^{t_{f}}\left(t_{f}-\xi\right) \mathbf{G}\left\{\mathbf{r}_{I}\left[\mathbf{r}_{I}(t), \mathbf{v}_{R}(t), \xi\right]\right\} d \xi
\end{aligned}
$$

The preceding relation may be solved approximately for the guidance problem, which is beyond the scope of the present study.

\section{CONCEPTUAL GUIDANCE LAWS}

It is necessary to determine the effective direction of interceptor thrust vector for short-to-super range exoatmospheric intercept problem. The thrust direction is determined by a conceptual guidance law (GL). After calculation of the effective thrust direction, a steering law is needed to convert the errors into commended body rates. The interceptor is assumed to be non-throttleable with thrust cutoff capability. The conceptual guidance laws are, here, categorized in 5 main classes, namely, guidance laws based on ZEM, guidance laws based on linearized ZEM, guidance laws based on generalized velocityto-be-gained, guidance laws based on constant direction for velocity-to-be-gained, and optimal solution.

\section{GUIDANCE LAWS BASED ON ZERO-EFFORT MISS}

In this case, the thrust acceleration is assumed to be applied perfectly in the direction of ZEM. Three guidance schemes may be considered regarding to 2 definitions of ZEM and also the component of ZEM perpendicular to LOS as follows:

$$
\begin{array}{ll}
\mathbf{a}_{t h}=\left|\mathbf{a}_{t h}\right| \mathbf{e}_{\text {ZEM }} & \text { for }|\mathbf{Z E M}|>\varepsilon \\
\mathbf{a}_{t h}=\left|\mathbf{a}_{t h}\right| \mathbf{e}_{\text {ZEM }_{\text {min }}} & \text { for }\left|\mathbf{Z E M}_{\text {min }}\right|>\varepsilon \\
\mathbf{a}_{t h}=\left|\mathbf{a}_{t h}\right| \mathbf{e}_{\text {ZEM }_{\text {PLOS }}} & \text { for }\left|\mathbf{Z E M}_{\mathrm{PLOS}}\right|>\varepsilon
\end{array}
$$

where: $\mathbf{e}_{\mathrm{ZEM}}, \mathbf{e}_{\mathrm{ZEM}_{\text {min }}}$ and $\mathbf{e}_{\mathrm{ZEM}_{\mathrm{PLOS}}}$ are the unit vectors along $\mathbf{Z E M}, \mathbf{Z E M}_{\text {min }}$, and $\mathbf{Z E M}_{\text {PLOS }}$, respectively; $\varepsilon$ is an allowable miss distance, determined from practical considerations.

In each guidance law, the powered phase of flight is terminated when its corresponding | ZEM | becomes equal or less than $\varepsilon$ and then it is followed by a coasting phase until intercept. The component of ZEM normal to LOS is calculated by:

$$
\mathbf{Z E M}_{\mathrm{PLOS}}=\mathbf{Z E M}-\left(\mathrm{ZEM} \cdot \mathbf{e}_{r}\right) \mathbf{e}_{r}
$$

where: $\mathbf{e}_{r}=\mathbf{r} / r$ is the unit vector along LOS $(r=|\mathbf{r}|)$.

To calculate the time-to-go until intercept in $\mathbf{Z E M}_{\text {PLOS }}$ relation, the component of ZEM along LOS is imposed to be zero. For a special case of a free-falling target in a flat-Earth model, the relation $t_{g_{o}}=-r / \dot{r}$ zeros out the LOS component of ZEM.

\section{GUIDANCE LAWS BASED ON LINEARIZED ZERO-EFFORT MISS}

Here, conceptual guidance laws are given using linearized relations for the problem. The first is similar to Eq. 31, but with linearized relation for ZEM, that is,

$$
\mathbf{a}_{t h}=\left|\mathbf{a}_{t h}\right| \mathbf{e}_{\mathrm{ZEM}_{\text {Lin }}} \text { for }\left|\mathbf{Z E M}_{\text {Lin }}\right|>\varepsilon
$$


where: $\mathbf{e}_{\mathrm{ZEM}_{\mathrm{Lin}}}$ is the unit vector along $\mathbf{Z E M}_{\mathrm{Lin}}$.

The second conceptual guidance is based on the linear optimal guidance law (OGL) obtained by Deihoul and Massoumnia (2003). Their linearized OGL may be expressed in the following form:

$$
\mathbf{U}_{\text {Lin }}=\mathbf{M}\left(t_{b g o}, t_{g o}, r_{T}, r_{I}\right) \mathbf{Z E M}_{\text {Lin }}
$$

where: $\mathbf{U}_{\mathrm{Lin}}$ is the optimal thrust vector for a throttleable rocket motor; $\mathbf{M}$ is a $3 \times 3$ matrix that causes the thrust vector to deviate from $\mathbf{Z E M}_{\text {Lin }}$ direction and change its magnitude as well; $t_{b g o}$ is the time-to-go until burnout.

The relation of matrix $\mathbf{M}$ is available in Deihoul and Massoumnia (2003). The thrust direction in the second conceptual guidance is applied in the direction of $\mathbf{U}_{\text {Lin }}$ for non-throttleable rocket motors as follows:

$$
\mathbf{a}_{t h}=\left|\mathbf{a}_{t h}\right| \mathbf{e}_{\mathbf{U}_{\text {Lin }}} \text { for }\left|\mathbf{Z E M}_{\text {Lin }}\right|>\varepsilon
$$

To compare the performances of the guidance schemes precisely, a third relation based on the exact calculation of ZEM in a spherical-Earth model is written as follows:

$$
\mathbf{a}_{t h}=\left|\mathbf{a}_{t h}\right| \mathbf{e}_{\mathbf{U}} \quad \text { for }|\mathbf{Z E M}|>\varepsilon
$$

where: $\mathbf{e}_{\mathbf{U}}$ is the unit vector along $\mathbf{U}$ calculated by:

$$
\mathbf{U}=\mathbf{M}\left(t_{b g o}, t_{g o}, r_{T}, r_{I}\right) \mathbf{Z E M}
$$

where ZEM is computed numerically.

\section{GUIDANCE LAW BASED ON VELOCITY-TO-BE-GAINED}

In space missions a class of guidance laws is based on required velocity with the desired thrust acceleration along the velocityto-be-gained. The required velocity concept and velocity-to-begained can be generalized for interception of a moving target, as mentioned before. The conceptual guidance law is then given by:

$$
\mathbf{a}_{t h}=\left|\mathbf{a}_{t h}\right| \mathbf{e}_{v_{g}} \text { for }\left|\mathbf{v}_{g}\right|>\varepsilon_{v}
$$

where: $\mathbf{e}_{v_{g}}$ is the unit vector along the velocity-to-be-gained; $\boldsymbol{\varepsilon}_{v}$ is an allowable velocity-to-be-gained error.

It is worth noting that the preceding conceptual guidance law is equivalent to Eq. 31 for constant gravity assumption as is evident from Eq. 29.

\section{GUIDANCE BASED ON CONSTANT DIRECTION OF VELOCITY-TO-BE-GAINED}

An effective direction of thrust acceleration in space missions is obtained by satisfying the relation $\dot{\mathbf{v}}_{g} \times \mathbf{v}_{g}=\mathbf{0}$ as follows (Battin 1999):

$$
\mathbf{a}_{t h}=\mathbf{b}+\left(q-\mathbf{b} \cdot \mathbf{e}_{v_{g}}\right) \mathbf{e}_{v_{g}}
$$

where:

$$
\begin{aligned}
& q=\sqrt{\left|\mathbf{a}_{t h}\right|^{2}-|\mathbf{b}|^{2}+\left(\mathbf{b} \cdot \mathbf{e}_{v_{g}}\right)^{2}} \\
& \mathbf{b}=-\mathbf{Q} \mathbf{v}_{g}, \mathbf{Q}=\frac{\partial \mathbf{v}_{R}}{\partial \mathbf{r}_{I}}
\end{aligned}
$$

This conceptual guidance law causes the direction of velocityto-be-gained to be fixed in inertial space.

\section{RESULTS AND DISCUSSION}

To compare the performance of conceptual guidance laws, a nonlinear flight simulation is utilized. The interceptor and its target are taken as particles in vertical planar motion with perfect dynamics, i.e. the thrust acceleration is assumed to be exactly in the desired direction of computed thrust direction, without any error or delay.

First, guidance laws (Eqs. 31 - 33) with different ZEM definitions, i.e. $\mathbf{Z E M}, \mathbf{Z E M}_{\text {min }}$, and also the normal component of ZEM, ZEM $_{\text {PLOS }}$, are compared in a flat-Earth model with constant gravity. The interceptor is located at origin $(0,0)$ with $a_{t h}=50 \mathrm{~m} / \mathrm{s}^{2}$ $\left(a_{t h}=\left|\mathbf{a}_{t h}\right|\right)$. The fuel consumption, $\Delta V=\left|\mathbf{a}_{t h}\right| t_{c o}$, of the mentioned conceptual guidance laws are shown in Figs. $2-4$, where $t_{c o}$ is the thrust cut-off time, applied when $|\mathbf{Z E M}| \leq \varepsilon$. In these figures, the solid lines indicate the fuel consumption when the thrust vector is applied along ZEM direction. The circle and square signs indicate the values of fuel consumption when the thrust direction is applied along $\mathbf{Z E M}_{\text {min }}$ and $\mathbf{Z E M}_{\text {PLOS' }}$ respectively, for their corresponding resulted final times. In Fig. 2, the fuel consumption is depicted versus predetermined final time for 2 cases of an initial 0 velocity, $\mathrm{v}_{\mathrm{I}}(0)=0$, and a vertical velocity of $\mathrm{v}_{\mathrm{I}_{z}}(0)=1 \mathrm{~km} / \mathrm{s}$ for a stationary target at $\mathbf{r}_{T}=\left[\begin{array}{ll}500 & 0\end{array}\right]^{\mathrm{T}} \mathrm{km}$. As seen in Fig. 2, the fuel consumption depends on the value of the final time when the thrust vector is applied along ZEM. 
Also, the minimum fuel consumption is not occurred necessarily when $\mathbf{Z E M}_{\text {min }}$ is utilized; however, it may occur for a special case. It should be noted that an appropriate final time (or timeto-go until intercept) is estimated in practice.

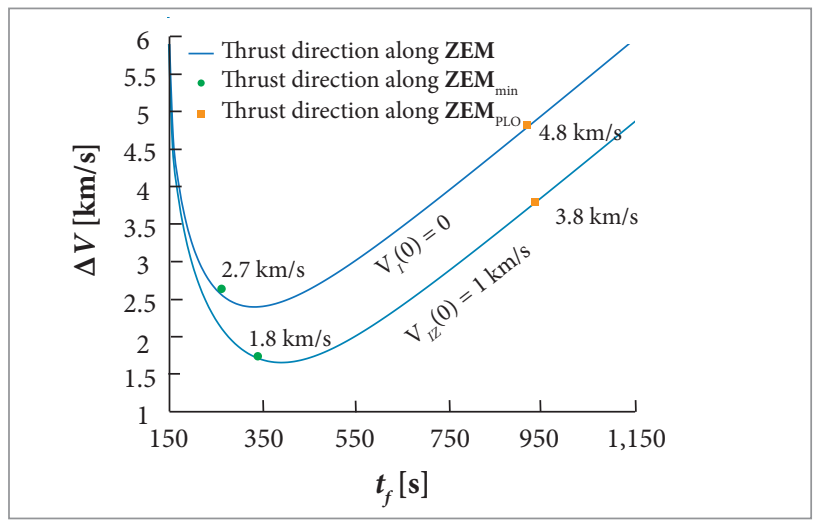

Figure 2. Fuel consumption versus final time for interception of a stationary target in flat-Earth model.

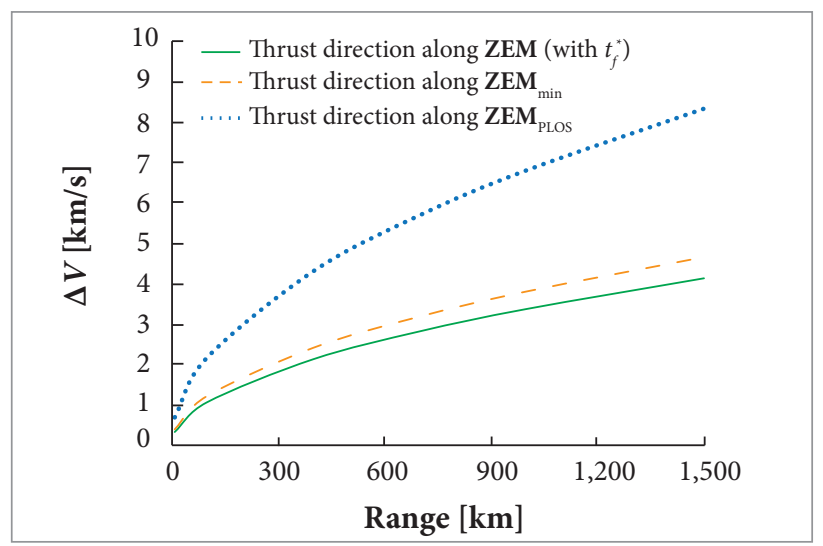

Figure 3. Fuel consumption versus range for interception of a stationary target in flat-Earth model.

(a)

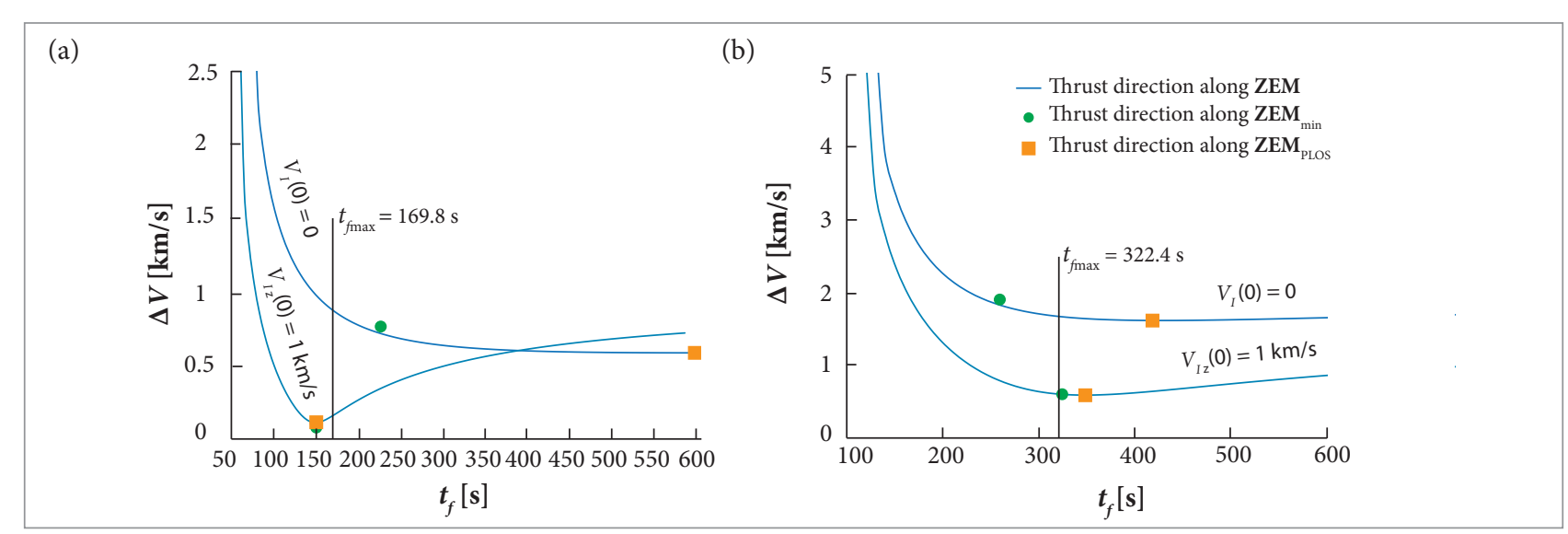

In order to investigate more precisely, the fuel consumption is drawn versus range in Fig. 3 for the 3 mentioned guidance schemes when the interceptor launches from rest. Other initial values and parameters are similar to Fig. 2. As shown in Figs. 2 and 3 , the fuel consumption is highly increased using guidance

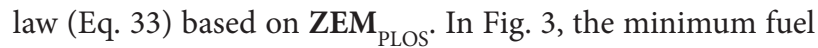
consumption for the case of thrust direction along ZEM is computed by setting the optimum value, $t_{f}^{*}$, for intercept time. In other words, $t_{f}^{*}$ is the intercept time for minimum $\Delta V$ when the thrust acceleration is imposed along ZEM. The value of $\varepsilon$ is, here, chosen as $1 \mathrm{~m}$ due to numerical errors.

In the next step, the performances of the conceptual guidance laws are compared in Figs. $4 \mathrm{a}$ and $4 \mathrm{~b}$ for a free-falling target at an initial altitude of $100 \mathrm{~km}$, having a minimum required velocity to hit the origin in a flat-Earth model. Initial values and parameters are similar to Fig. 2, except target position. The target range is taken 100 and $500 \mathrm{~km}$ for Figs. $4 \mathrm{a}$ and $4 \mathrm{~b}$, respectively. A vertical solid line has been drawn for each of Figs. $4 \mathrm{a}$ and $4 \mathrm{~b}$, showing the maximum possible final time $\left(t_{f_{\max }}\right)$, i.e. the time of hitting origin $(0,0)$ by the free-falling target. The region at the right-hand side of this vertical line is not acceptable, because the interception of a free-falling target occurs behind the origin in negative altitudes. This is an important difference between the two cases of stationary and free-falling targets.

According to our analysis for a flat-Earth model, the capture criteria for the conceptual guidance law based on $\mathbf{Z E M}_{\text {PLOS }}$ are highly restricted comparing to the conceptual guidance law based on ZEM, at least, for non-throttleable rockets.

We are now to study the performance of conceptual guidance laws (Eqs. 31, 32, and 40) for a spherical-Earth model in Figs. 5 - 14 against stationary targets. The interceptor fires

Figure 4. Fuel consumption versus final time for interception of a free-falling target in flat-Earth model. (a) initial range = $100 \mathrm{~km}$; (b) initial range $=500 \mathrm{~km}$. 
from position $\mathbf{r}_{I_{0}}=\left[\begin{array}{ll}6400 & 0\end{array}\right]^{T} \mathrm{~km}$ with a velocity of $1 \mathrm{~km} / \mathrm{s}$ along near vertical horizon $\left(89^{\circ}\right)$ and $a_{t h}=50 \mathrm{~m} / \mathrm{s}^{2}$. The initial distance of target from the earth center is taken $r_{T}=6,500 \mathrm{~km}$.

First, the fuel consumptions of these guidance laws are plotted in Figs. $5 \mathrm{a} ; 5 \mathrm{~b}$ and $5 \mathrm{c}$ for a target at range angles of $10 ; 40$ and $70^{\circ}$, respectively. As seen in the figures, if the thrust direction is applied along the direction of $\mathbf{v}_{g}$, the fuel consumption is considerably reduced for long-range applications. To investigate the trajectory of the interceptor based on ZEM direction, four scenarios with different final times are selected. These points, namely, $S_{1} ; S_{2} ; S_{3}$ and $S_{4}$ are assigned in Fig. 5a for different specified final times when the range angle is $10^{\circ}$.

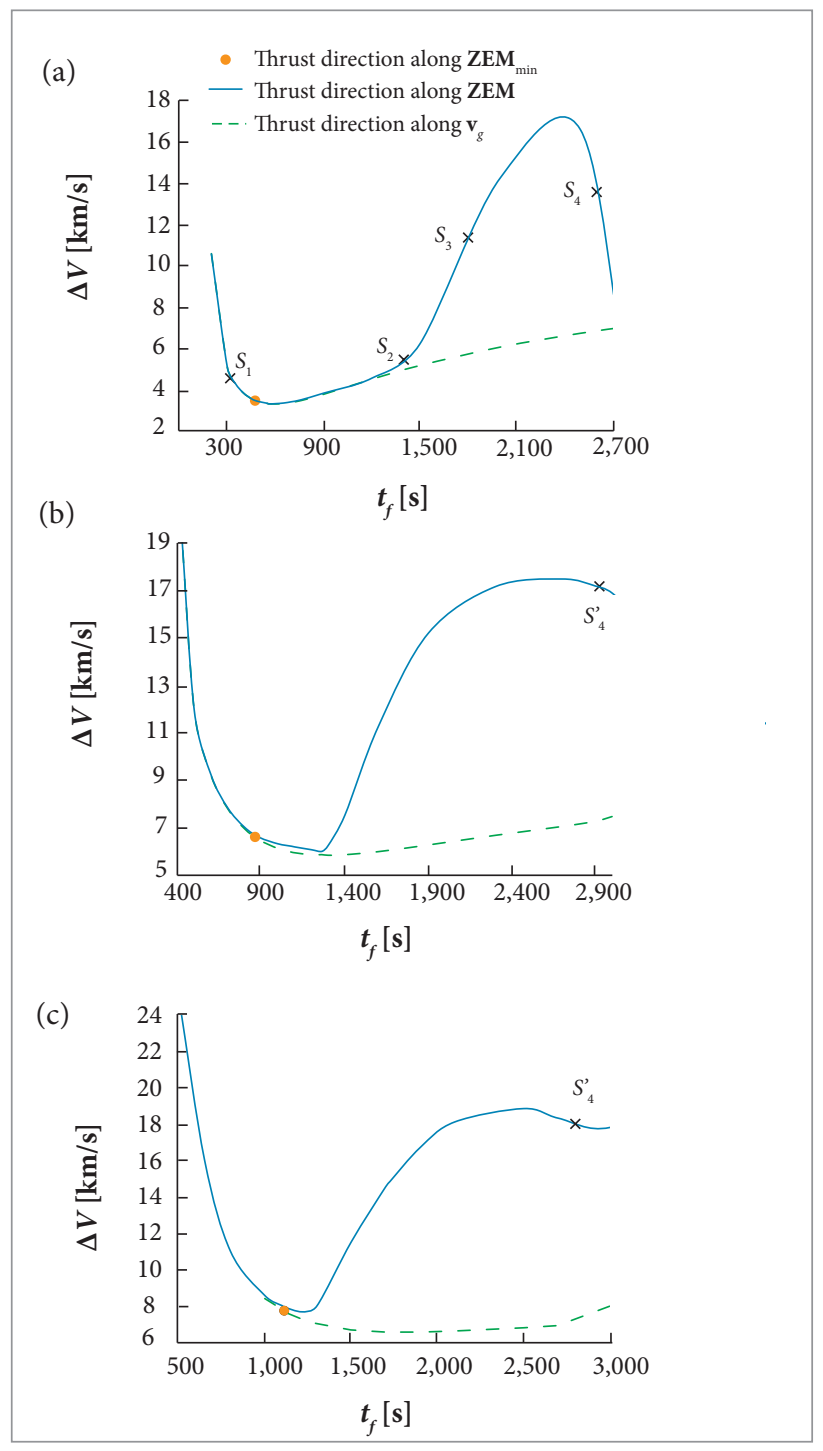

Figure 5. Fuel consumption versus final time for interception of a stationary target in spherical-Earth model. (a) range angle $=10^{\circ} ;$ (b) range angle $=40^{\circ}$; (c) range angle $=70^{\circ}$.
The typical interceptor trajectories of the mentioned scenarios are illustrated in Figs. 6a, 6b, 6c, and 6d for the points $S_{1}, S_{2}, S_{3}$, and $S_{4}$, respectively, and compared to interceptor trajectories based on $\mathbf{v}_{g}$ direction with the same final times. The interceptor trajectories based on ZEM and $\mathbf{v}_{g}$ are viewed by solid and dashed lines, respectively. The thrust cut-off time, $t_{c o}$, is also observed for each trajectory in the figures. In the case of ZEM-based trajectory, increasing total flight time causes an extra revolution of trajectory around the earth center, as shown in Fig. 6d. The maximum limit of total flight time to avoid this phenomena is assigned with $S_{4}$ in Fig. $5 \mathrm{a}$ and with $S_{4}{ }^{\prime}$ in Figs. 5b and $5 \mathrm{c}$ with different range angles. The typical interceptor trajectories with final times larger than that of $S_{4}$ ' in Figs. $5 b$ and $5 c$, are similar to Fig. 6d for ZEM-based trajectories. Moreover, Fig. 7 shows that the fuel consumption of $\mathbf{v}_{g}$-based guidance law is less sensitive to the estimation of total flight time. In this figure, the total flight time is considered about the total flight time for

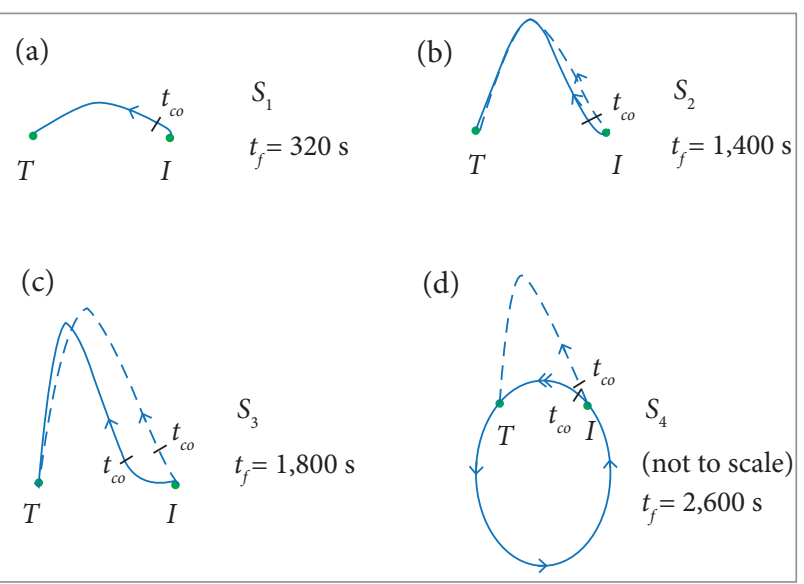

Figure 6. Typical interceptor trajectories for initial range angle $=10^{\circ}$ and different values of total flight times (solid line: thrust vector along ZEM direction; dashed line: thrust vector along $\mathbf{v}_{g}$ direction).

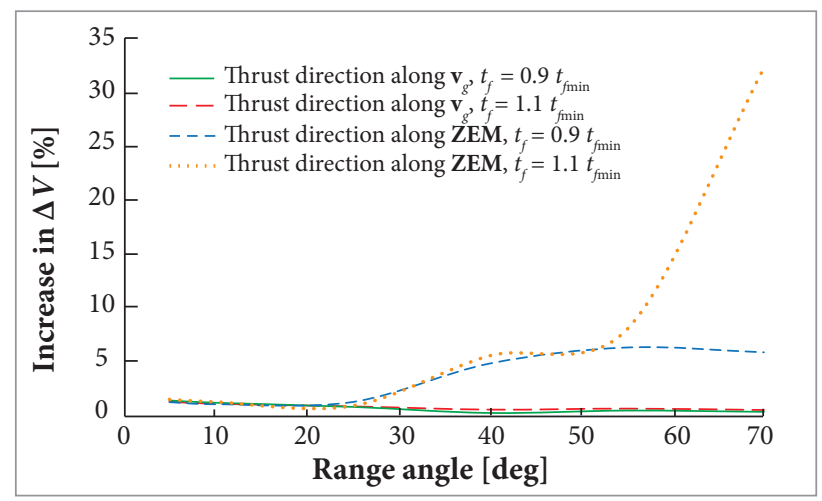

Figure 7. Increase in $\Delta V$ for guidance laws based on $\mathbf{v}_{g}$ and ZEM. 
minimum $\Delta V$ for each guidance scheme, and it is denoted by $t_{f_{\min }}$, which is obtained for each range.

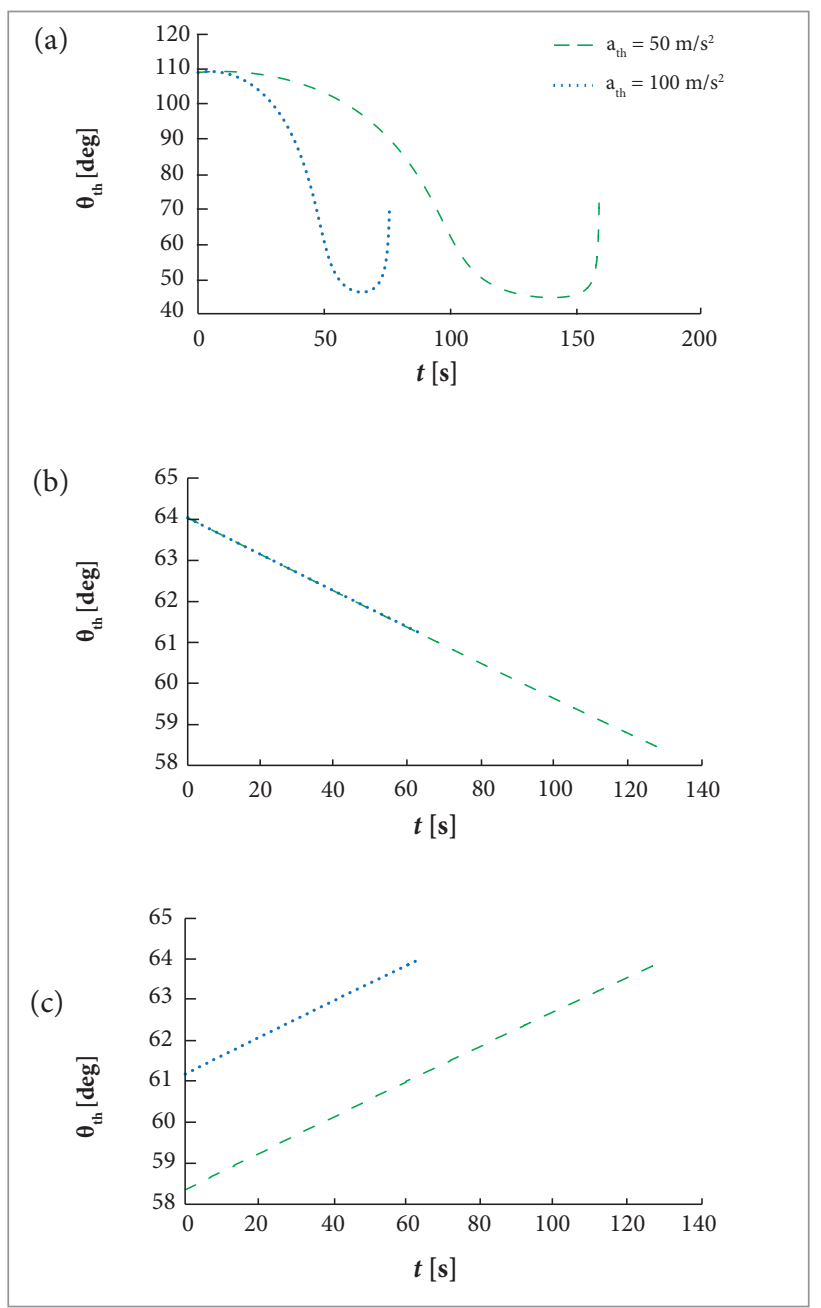

Figure 8. Thrust angle profiles with respect to the equatorial plane for interception of a stationary target for minimum energy orbit with initial range angle $=10^{\circ}$. (a) thrust vector along ZEM direction; (b) thrust vector along $\mathbf{v}_{g}$ direction; (c) constant $\mathbf{v}_{g}$ direction.

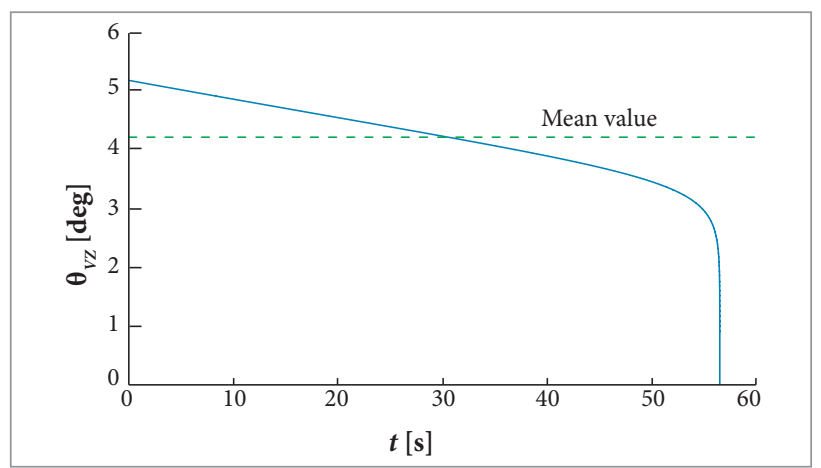

Figure 9. History of the angle between $\mathbf{v}_{q}$ and ZEM for thrust vector along 正M direction (minimum energy orbit with a range angle of $10^{\circ}$ ).
The total rotation of the thrust direction of conceptual guidance laws (Eqs. 31, 32, and 40) can be seen in Figs. 8a, $8 b$ and $8 c$ for different values of thrust acceleration of 50 and $100 \mathrm{~m} / \mathrm{s}^{2}$ when the range angle is $70^{\circ}$. The final times are chosen

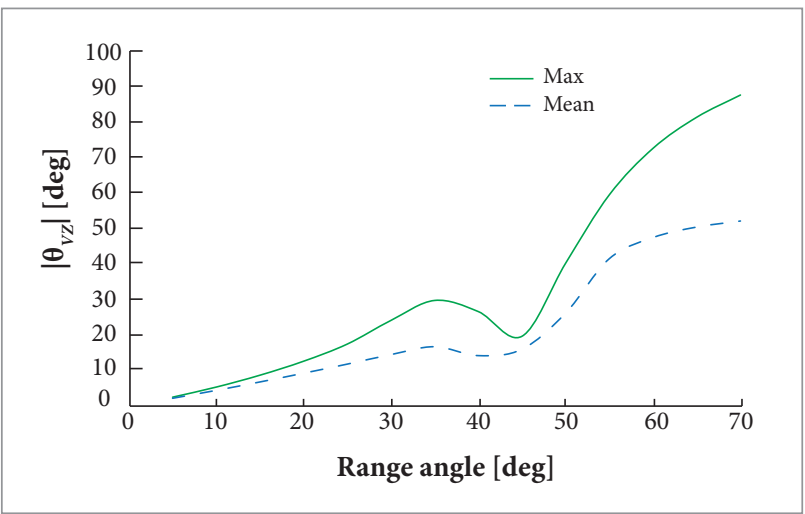

Figure 10. Max and mean of the absolute value of the angle between $\mathbf{v}_{g}$ and ZEM versus range angle for thrust vector along ZEM direction (minimum energy orbit).

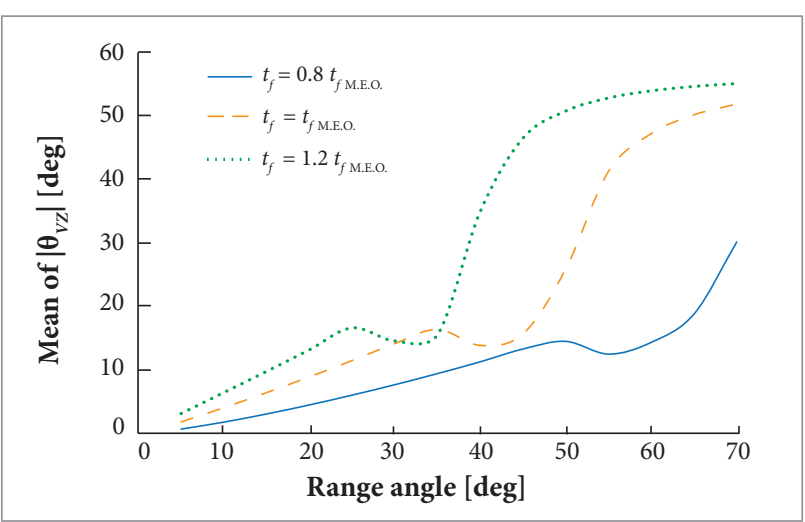

Figure 11. Mean of the absolute value of the angle between $v_{g}$ and ZEM versus range angle for thrust vector along ZEM direction with different total final times.

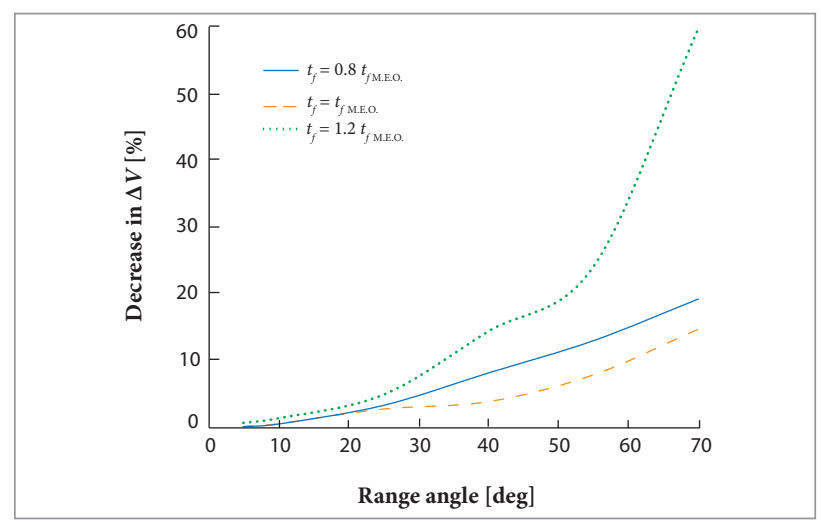

Figure 12. Decrease in $\Delta V$ for $\mathbf{v}_{g}$ - based guidance with respect to ZEM-based guidance for minimum and non-minimum energy orbit. 
for minimum energy orbit. Other parameters and initial values are similar to Fig. 5. In these figures, $\theta_{\text {th }}$ is the angle of the thrust acceleration with respect to the equatorial plane. As can be seen in these figures, the guidance law based on ZEM has a larger total rotation of the thrust vector than those of the two guidance laws based on $\mathbf{v}_{g}$. Also, it is observed that the rate of change of $\theta_{t h}$ is nearly constant for the two guidance laws based on $\mathbf{v}_{g}$. This property phenomena can be used for implementation of the guidance laws based on $\mathbf{v}_{g}$.

An important question comes from the implementation point of view: what is the typical value of the angle between ZEM and $\mathbf{v}_{g}$ ? If this value is large enough to overwhelm the control system tracking error and estimation error of required velocity in the presence of target tracking error, the performance study of the guidance law can go ahead for this purpose.

First, the angle between $\mathbf{v}_{g}$ and ZEM, denoted by $\theta_{V Z}$, is depicted in Fig. 9 versus time for an interceptor when its thrust acceleration is applied along $\mathbf{Z E M}$ for a range angle of $10^{\circ}$. The maximum and the mean of $\left|\theta_{V Z}\right|, \int_{o}^{t_{c o}}\left|\theta_{V Z}\right| d t / t_{c o}$, are observed by solid and dashed lines, respectively. The maximum and mean

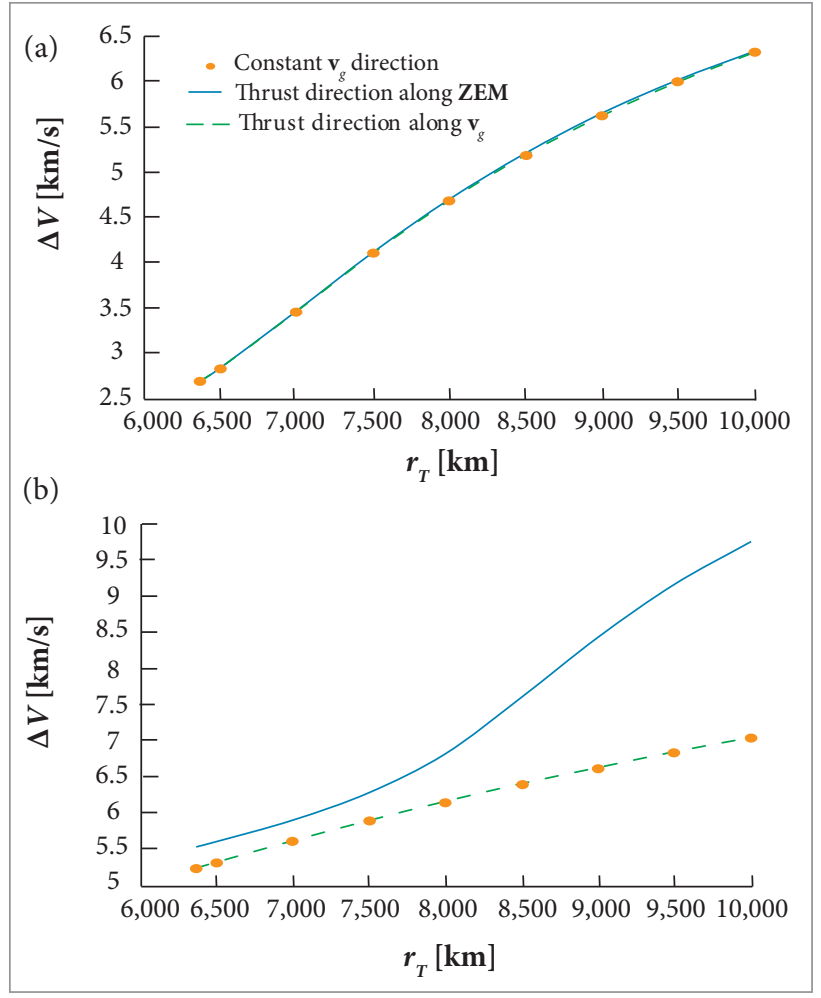

Figure 13. Fuel consumption versus target radial position for interception of a stationary target in spherical-Earth model. (a) range angle $=10^{\circ}$; (b) range angle $=40^{\circ}$. values of $\left|\theta_{V Z}\right|$ are shown in Fig. 10 versus range angle. The final times in Figs. 9 and 10 are chosen for minimum energy orbit, i.e. minimizing the required velocity. To investigate more precisely, the mean value of $\left|\theta_{V Z}\right|$ is plotted in Fig. 11 versus range angle for a deviation of $\pm 20 \%$ with respect to the final time of minimum energy orbit. As expected, the values of $\theta_{V Z}$ are large enough to overcome noisy measurements and control system tracking error for medium-to-super range applications. The effect of this deviation on $\Delta V$ can be viewed in Fig. 12 where the comparison is made with respect to the fuel consumption of the ZEM-based guidance law.

The effect of target altitude is investigated in Fig. 13. First, the fuel consumption is plotted versus target radial position for a range angle of $10^{\circ}$ for the minimum energy orbit. As can also be seen in Fig. 5a, there is little difference between the fuel consumptions of $\mathbf{v}_{g}$ - and ZEM-based guidance laws. This difference is increased by increasing the range angle. For example, the difference is shown in Fig. 13b for the range angle of $40^{\circ}$. Moreover, the maximum value of the angle between $\mathbf{v}_{g}$ and ZEM versus target radial position can be viewed in Figs. $14 \mathrm{a}$ and $14 \mathrm{~b}$ for minimum and non-minimum energy orbits,
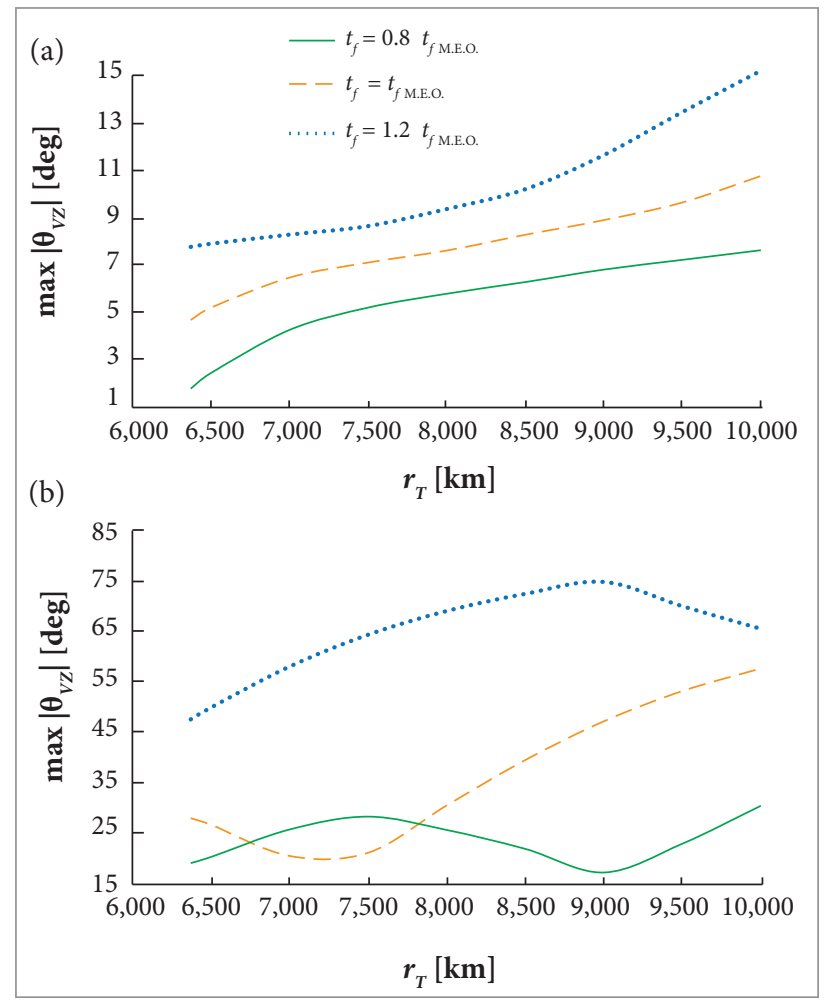

Figure 14. Max of the absolute value of the angle between $\mathbf{v}_{g}$ and ZEM versus target radial position for thrust vector along ZEM direction. (a) range angle $=10^{\circ}$; (b) range angle $=40^{\circ}$. 
e.g. $t_{f}=(1 \pm 0.2) t_{f \text { M.E.O. }}$, respectively, where M.E.O. is the mean energy orbit.

After a preliminary study of conceptual guidance laws for flat-Earth model and also stationary targets in spherical-Earth model, we focus on free-falling targets in the case of sphericalEarth model. The interceptor initial position, velocity, and acceleration due to thrust is taken similar to the studied case of spherical-Earth model with stationary targets.

First, the guidance law based on linearized ZEM, Eq. 26, is compared to the guidance based on the direction computed by linear optimal guidance law (Eq. 36) as obtained by Deihoul and Massuomnia (2003). The miss distance and fuel consumption of the two guidance schemes are plotted in Figs. 15 and 16 versus final time for a target at a range angle of $40^{\circ}$, having the required velocity of minimum energy to hit the initial position of the interceptor. The initial distance of target from the Earth center is taken $r_{T}=6,500 \mathrm{~km}$. These 2 guidance laws produce nearly the same results; however, the little differences in the results cannot appear properly in Figs. 15 and 16 because of the

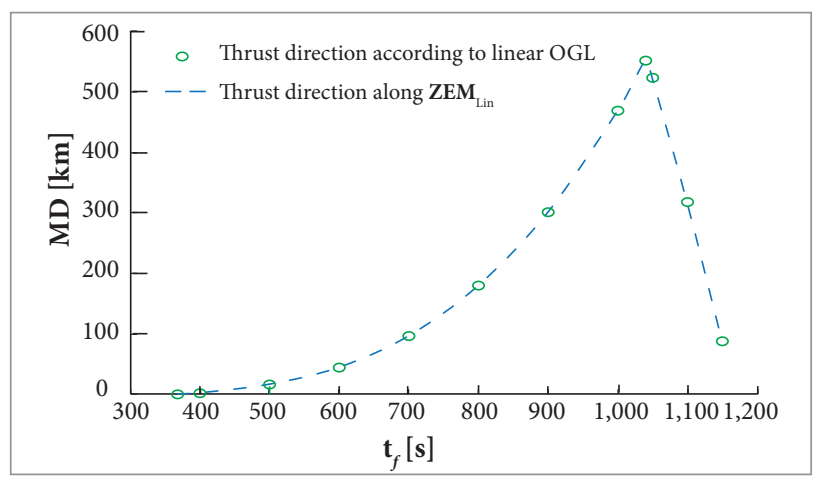

Figure 15. Miss distance (MD) versus final time for interception of a free-falling target in spherical-Earth model using linearized ZEM relation (initial range angle $=40^{\circ}$ ).

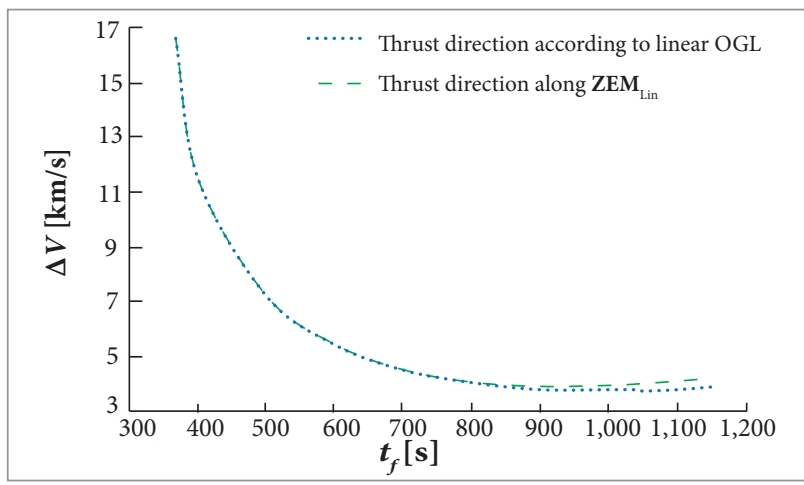

Figure 16. Fuel consumption versus final time for interception of a free-falling target in spherical-Earth model (initial range angle $=40^{\circ}$ ). scale of these figures. These results are obtained by setting an optimized value of $t_{b}=60 \mathrm{~s}$ in Eq. 37 for the gain matrix $\mathbf{M}$. This analysis turns out the gain matrix $\mathbf{M}$ do not give a significant improvement on the performance of the ZEM-based guidance schemes for an initial range angle less than $40^{\circ}$.

To study more precisely, the initial distance of the target from the earth center increases to $7,500 \mathrm{~km}$. First, the value of $t_{b}$ is chosen $100 \mathrm{~s}$ by the performance analysis based on Fig. 17 for an initial range angle of $90^{\circ}$ with 3 different values of total final times. In the next step, the fuel consumptions of four conceptual guidance laws, i.e. guidance laws based on $\mathbf{Z E M}_{\text {min }}$, based on ZEM, based on the direction of linear optimal problem, and based on velocity-to-be-gained are compared together. For this purpose, the fuel consumption is depicted versus final time in Figs. $18 \mathrm{a} ; 18 \mathrm{~b}$ and $18 \mathrm{c}$ for 3 different initial range angles of $60^{\circ}$; $90^{\circ}$ and $120^{\circ}$, respectively. The value of $t_{b}$ is optimized for each range angle. Other parameters and initial values are taken as before. For a typical range comparison, the interceptor travels $29.7^{\circ}(3,301 \mathrm{~km})$ for an initial range angle of $90^{\circ}(10,002 \mathrm{~km})$ as plotted in Fig. 19 for a total flight time of $1,600 \mathrm{~s}$. The thrust direction along the velocity-to-be-gained produces less fuel consumption among the other mentioned guidance laws as shown in the Fig. 18. For short range applications, the angle between ZEM and velocity-to-be-gained is negligible as shown in Fig. 20. The angle between the 2 directions increases by increasing the initial range angle. The effect of matrix gain of $\mathbf{M}$ is also increased by increasing the range angle, but for these ranges the direction of the velocity-to-be-gained produces a considerable decrease on fuel consumption. Besides, the estimation error of time-to-go for guidance laws based on velocity-to-be-gained has less sensitivity than that of ZEM-based guidance schemes.

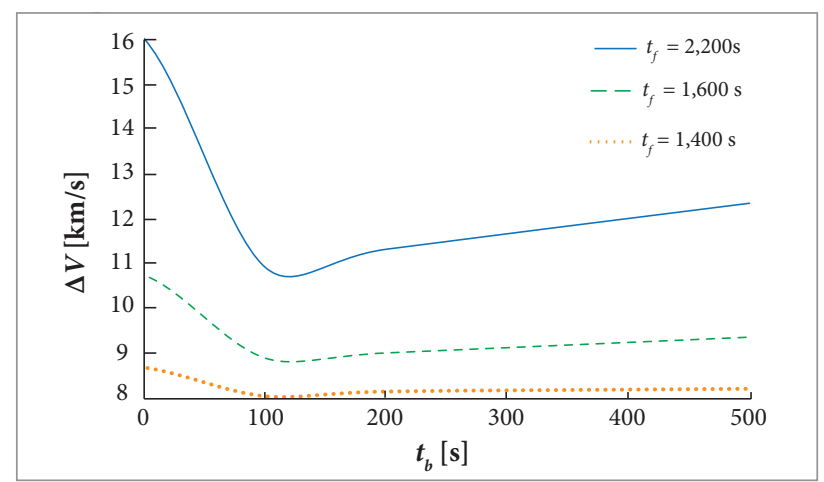

Figure 17. Fuel consumption versus burnout time for interception of a free-falling target in spherical-Earth model, when thrust vector is along linearized ZEM direction (initial range angle $=90^{\circ}$ ) 
The angle of thrust direction with respect to the equatorial plane is plotted in Fig. 21 versus time for 3 guidance laws. As can be seen, there is a little difference between the velocity-tobe-gained direction and the direction of guidance laws based on constant $\mathbf{v}_{g}$ direction. The maximum difference for an initial range angle of $70^{\circ}$ is about $5.26^{\circ}$. In addition, the rate of change of thrust direction for guidance laws based on velocity-to-begained are very small comparing to that of the guidance laws based on ZEM. To investigate more precisely the difference between the thrust angles under the 2 conceptual guidance laws based on velocity-to-be-gained, $\theta_{B V}$, is shown in Fig. 22 in three forms, i.e. max of $\left|\theta_{B V}\right|$, mean of $\left|\theta_{B V}\right|$, and mean of

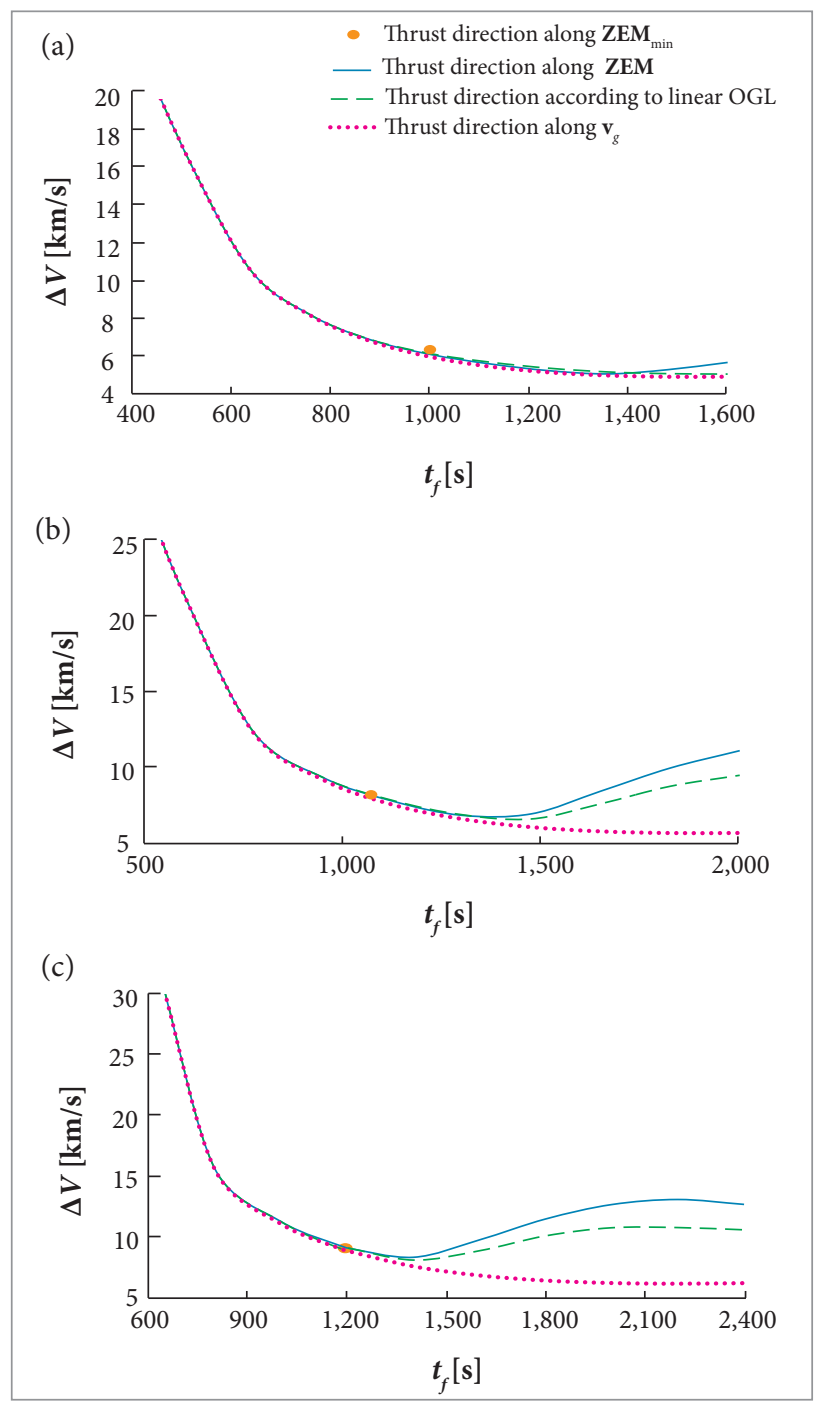

Figure 18. Compassion of fuel consumption of four conceptual guidance laws for interception of a free-falling target in spherical-Earth model. (a) initial range angle $=60^{\circ}$; (b) initial range angle $=90^{\circ}$; (c) initial range angle $=120^{\circ}$.
$\theta_{B V}$ for a minimum energy orbit and a stationary target. Initial values for interceptor and its target are the same as Figs. 5 - 7 . As seen in Fig. 22, the mean value of $\theta_{B V}$ is $1.11^{\circ}$ for an initial

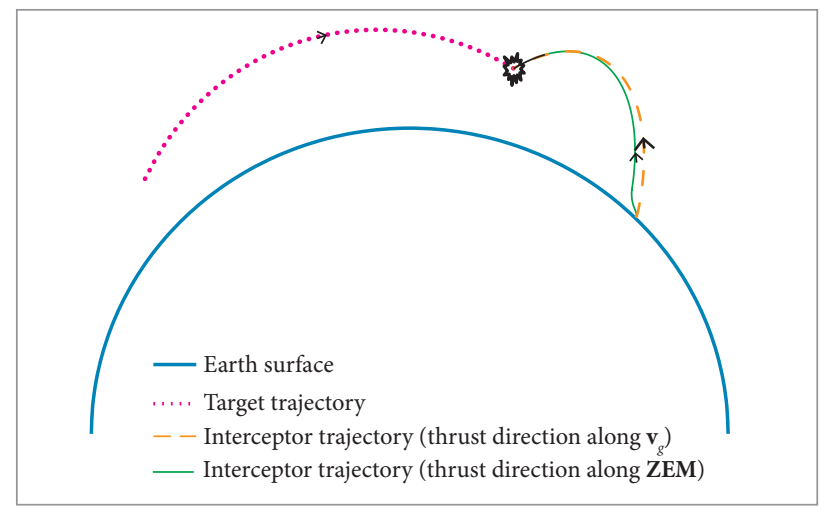

Figure 19. Interceptor and its target trajectories for initial range angle of $90^{\circ}$ and total flight time of 1,600 s.

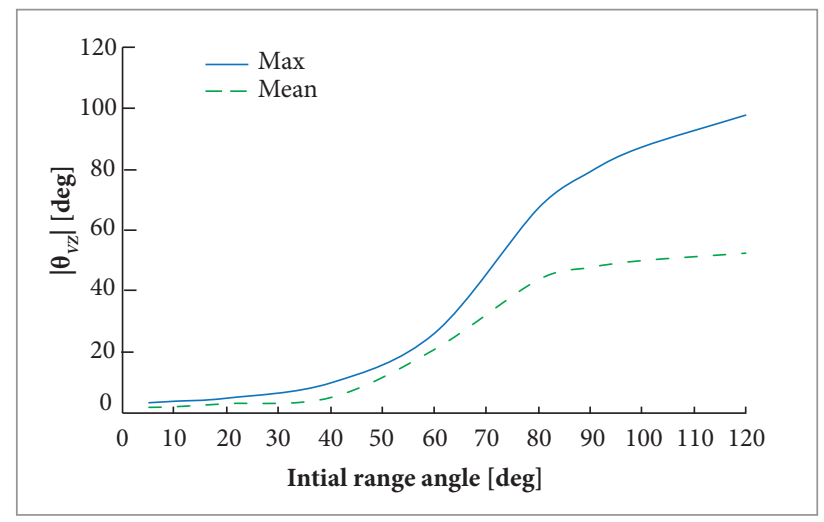

Figure 20. Maximum and mean of the absolute value of the angle between $\mathbf{v}_{g}$ and ZEM versus initial range angle for interception of a free-falling target (thrust vector along ZEM direction, minimum energy orbit].

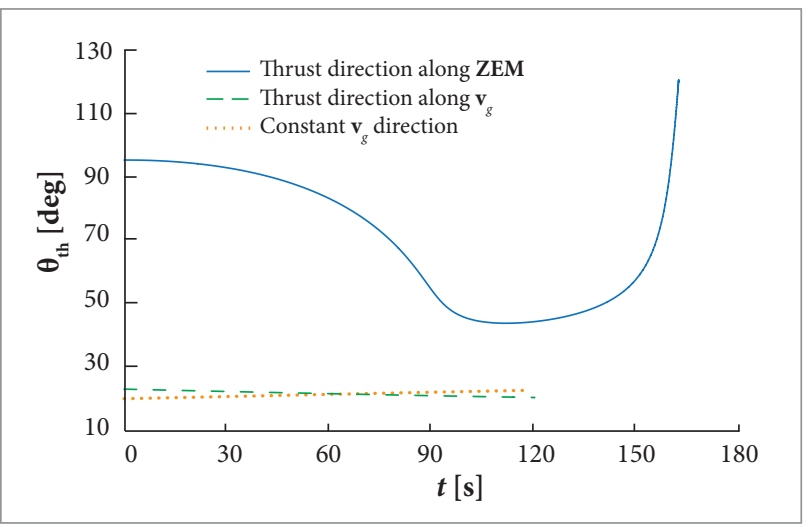

Figure 21. Comparison of thrust angle with respect to the equatorial plane for 3 conceptual guidance laws (initial range angle $=70^{\circ} ; a_{\text {th }}=50 \mathrm{~m} / \mathrm{s}^{2}$ ]. 
range angle of $70^{\circ}$. Their fuel consumptions are compared in Fig. 23 versus initial range angle for minimum and nonminimum energy orbit. For example, the guidance based on the constant $\mathbf{v}_{g}$ direction causes a decrease of $0.32 \%$ in fuel consumption for minimum energy orbit with respect to the guidance law based on $\mathbf{v}_{g}$ direction for a range angle of $180^{\circ}$. As seen in Fig. 22, below the initial range angle of $10.3^{\circ}$, $\max$ $\left|\theta_{B V}\right|$ is less than $1^{\circ}$. However, improving the performance of a guidance law is always of interest by a modified formulation without any additional hardware or extra cost. Fortunately, several iterative and approximate methods are available in literature to calculate the required velocity and Q-matrix for Lambert's problem.

For space missions, Sokkappa (1966) developed a near optimal guidance in closed-loop for throttleable spacecraft assuming constant Q-matrix; however, the Q-matrix was updated for onboard computation. Our simulation results show that the maximum difference between the thrust angles of Sokkappa's

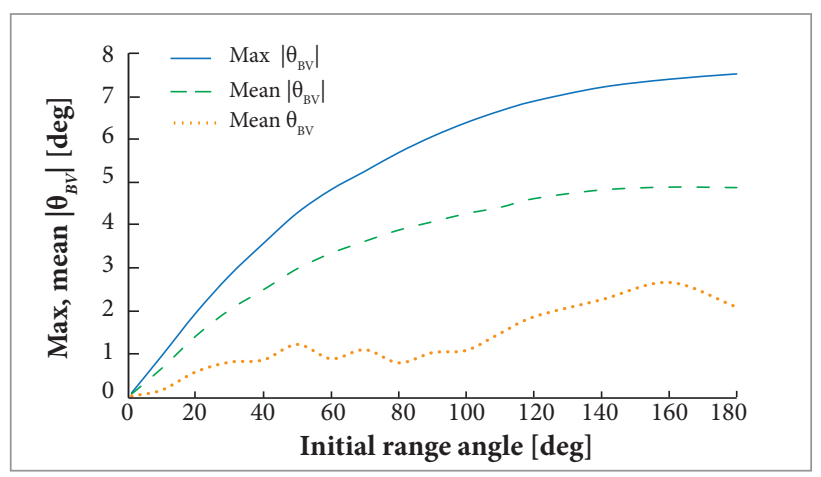

Figure 22. Maximum and mean of the absolute value, and mean value of the angle between the thrust direction along $\mathbf{v}_{g}$ and constant $\mathbf{v}_{g}$ direction versus range angle for interception of a stationary target for minimum energy orbit.

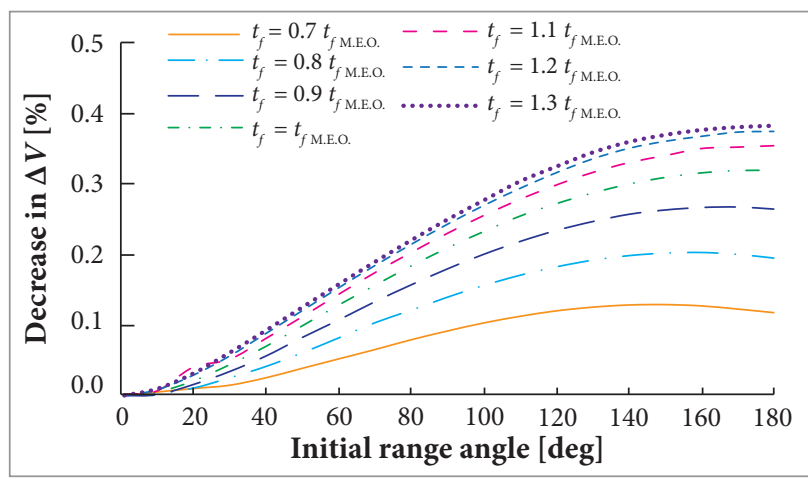

Figure 23. The percentage decrease in $\Delta V$ for the guidance law based on constant $\mathbf{v}_{g}$ direction with respect to $\mathbf{v}_{g}$ direction for minimum and non-minimum energy orbit. solution and guidance based on constant $\mathbf{v}_{g}$ direction is less than $0.5^{\circ}$ when the initial range angle is less than $180^{\circ}$ (the initial values and parameters are similar to Fig. 22). In addition, Sokkappa compared his optimal solution with the guidance law based on constant $\mathbf{v}_{g}$ direction for a case of injection from an earth orbit of 100 nautical miles to pass through an inertial point of 180,000 nautical miles. The guidance law based on constant $\mathbf{v}_{g}$ direction produces $\Delta \mathrm{V}=3,328.6 \mathrm{~m} / \mathrm{s}$, whereas Sokkappa's solution has a $34.2 \mathrm{~m} / \mathrm{s}$ decrease in $\Delta \mathrm{V}$. Also, the performance of guidance law based on constant $\mathbf{v}_{g}$ direction and numerical optimal solution was compared by Circi (2004) for a satellite launch vehicle. The optimum solution has a decrease of $39 \mathrm{~kg}$ for a payload mass of $1,734 \mathrm{~kg}$ under the guidance law based on constant $\mathbf{v}_{g}$ direction for a perigee of $150 \mathrm{~km}$.

It is worth noting that the guidance laws based on velocityto-be-gained are applicable provided that a required velocity can be defined. For example, a required velocity cannot be defined for a fixed-final-time problem when final position and velocity vectors are both constrained. The impact angle of the conceptual guidance schemes is not considered in this investigation. It may be accomplished using appropriate choice of final time.

\section{CONCLUSIONS}

This study suggests the effective thrust direction of an exoatmospheric interceptor for interception of short-tosuper range moving targets with final position constraint. This has accomplished using a comprehensive study on conceptual guidance laws with stationary, moving, and freefalling targets. The first class of guidance law is based on ZEM. Three guidance schemes are considered in the first class, regarding to 2 definitions of ZEM and the component of ZEM perpendicular to line-of-sight. The capture criteria of the guidance scheme based on the perpendicular component of ZEM are highly restricted for non-throttleable rockets. The second class of conceptual guidance laws are based on linearized formulation, i.e. linearized ZEM and linear optimal control theory for throttleable rockets when its computed thrust direction is applied to non-throttleable rockets. The third class of guidance laws is based on the generalized required velocity and generalized velocity-to-be-gained. Two guidance schemes are considered in the third class. In the first scheme, the thrust acceleration is applied along the velocity-to-be-gained vector, 
whereas the second scheme tries to avoid the rotation of the velocity-to-be-gained in an inertial space.

The suggested direction of thrust acceleration is along the generalized velocity-to-be-gained, defined based on the generalized required velocity for interception of moving targets. For short-range application, the same results have been achieved when the thrust acceleration applied in the direction of ZEM or in the direction of the generalized velocityto-be-gained. Increasing the range angle, the difference in performance is appeared. For long-range interception, the suggested thrust direction requires less amount of fuel rather than conceptual guidance laws based on ZEM or linearized formulations. The guidance performance is not improved as expected using a ZEM vector multiplied by a gain matrix to deviate the thrust direction from ZEM vector, as obtained in linearized optimal solutions.

If the intercept time is chosen a bit larger than the minimum energy orbit due to tactical consideration, e.g. salvo firing, adjustment of impact angle, etc., the suggested direction will have a significant fuel savings rather than guidance laws based on ZEM. Moreover, the performance of the suggested conceptual guidance law has less sensitive to the estimation error of final time. The guidance scheme based on constant velocity-to-be-gained direction may improve negligibly the fuel performance of the interceptor in the presence of noise for suborbital interception. Finally, the optimal solution does not give a better performance when the target position and velocity are contaminated by noise for suborbital interception such as a ballistic target; however, it improves the performance for a satellite launch vehicle and possibly for interception of orbital targets.

\section{AUTHOR'S CONTRIBUTION}

Mohamed-abadi MD performed the numerical solutions and prepared the figures. The idea, assumptions, classifications, and framework belong to Jalali-Naini SH, who wrote the manuscript. Both authors discussed the results and commented on the manuscript.

\section{REFERENCES}

Ahn J, Bang J, Lee SI (2015) Acceleration of zero-revolution Lambert's algorithms using table-based initialization. J Guid Control Dynam 38(2):335-342. doi: 10.2514/1.G000764

Arora N, Russell RP, Strange NJ, Ottesen D (2015) Partial derivatives of the solution to the Lambert boundary value problem. J Guid Control Dynam 38(9):1563-1572. doi: 10.2514/1. G001030

Battin RH (1999) An introduction to the mathematics and methods of astrodynamics. Revised edition. Reston: American Institute of Aeronautics and Astronautics.

Bryson AE, Ho YC (1975) Applied optimal control. New York: Hemisphere.

Chen FL, Xiao Y, Chen W (2010) Guidance based on velocity-tobe-gained surface for super-range exoatmospheric intercept. Acta Aeronautica et Astronautica Sinica 31(2):342-349. In Chinese.

Circi C (2004) Hybrid methods and Q-guidance for rocket performance optimization. Proc IME G J Aero Eng 218(5):353359. doi: 10.1243/0954410042467040

Deihoul AR (2003) Anti ballistic optimal midcourse guidance law (PhD thesis). Tehran: Sharif University of Technology. In Persian.

Deihoul AR, Massoumnia MA (2003) A near optimal midcourse guidance law based on spherical gravity. Scientia Iranica 10(4):436-442.

Feng C, Yelun X, Wanchun C (2009) Guidance based on zero effort miss for super-range exoatmospheric intercept. Acta Aeronautica et Astronautica Sinica 30(9):1583-1589. In Chinese.

Jalali-Naini SH (2004) Modern explicit guidance law for highorder dynamics. J Guid Control Dynam 27(5):918-922. doi: $10.2514 / 1.5902$

Jalali-Naini SH (2008) Generalization of zero-effort miss equations in atmospheric guidance laws with application to midcourse flight (PhD thesis). Tehran: Sharif University of Technology. In Persian.

Jalali-Naini SH, Pourtakdoust SH (2005) Modern midcourse guidance laws in the endoatmosphere. Proceedings of the AIAA Guidance, Navigation and Control Conference and Exhibit; San Francisco, USA.

Jalali-Naini SH, Pourtakdoust SH (2007) A unified approach to intercept guidance laws. Proceedings of the 6th Iranian Aerospace Society Conference; Tehran, Iran.

Li LG, Jing WX, Gao CS (2013) Design of midcourse trajectory for tactical ballistic missile intercept on the basis of zero effort miss. App Mech Mater 397-400:536-545. doi: 10.4028/www. scientific.net/AMM. 397-400.536

Martin FH (1965) Closed-loop near-optimum steering for a class of space missions (PhD thesis). Cambridge: Massachusetts Institute of Technology.

Martin FH (1966) Closed-loop near-optimum steering for a class of space missions. AIAA J 4(11):1920-1927. doi: 10.2514/3.3819 
Massoumnia MA (1995) Optimal midcourse guidance law for fixedinterval propulsive maneuvers. J Guid Control Dynam 18(3):465470. doi: $10.2514 / 3.21410$

Mohammad-abadi MD, Jalali-Naini SH (2016) Approximate solution of zero-effort-miss under gravitational acceleration inversely proportional to the cubic distance. Modares Mech Eng 16(4): 135-144. In Persian.

Newman B (1996) Strategic intercept midcourse guidance using modified zero effort miss steering. J Guid Control Dynam 19(1):107-112. doi: 10.2514/3.21586
Rusnak I, Meir L (1991) Modern guidance law for high-order autopilot. J Guid Control Dynam 14(5):1056-1058. doi: $10.2514 / 3.20749$

Sokkappa BG (1966) On optimal steering to achieve required velocity. Proceedings of the 16th International Astronautical Congress; Athens, Greece.

Zarchan P (2012) Tactical and strategic missile guidance. 6th edition. Reston: American Institute of Aeronautics and Astronautics. 